\title{
Impact of Target Coronary Artery Stenosis Severity Measured by Instantaneous Wave-Free Ratio on Bypassed Graft Patency
}

\author{
Almas Tolegenuly ${ }^{1}$, Arslan Mamedov ${ }^{2}$, Rimantas Benetis ${ }^{3}$ \\ ${ }^{1}$ Department of Cardiac, Thoracic and Vascular Surgery, Hospital of Lithuanian ${ }^{2}$ University of Health \\ Sciences Kauno Klinikos, Medical Academy, Lithuanian ${ }^{3}$ University of Health Sciences, Eivenių 2, LT- \\ 50009 Kaunas, Lithuania
}

\begin{abstract}
:
Background: This study aimed to assess the impact of the measurement of the degree of target coronary artery stenosis using the instantaneous wave-free ratio (iFR) on patency of attached grafts.

Materials and Methods: A total of 86 grafts were assessed by computed tomography angiography (CTA) after coronary artery bypass grafting $(\mathrm{CABG})$ in 24 patients with multivessel coronary artery disease (CAD). The iFR was evaluated for all target coronary arteries. The coronary artery stenoses were divided into three groups based on the iFR value: iFR < 0.86 (group 1); iFR 0.86-0.90 (group 2); and iFR > 0.90 (group 3).

Results: CTA was performed at $192 \pm 44$ days (range: 80-318 days). The correlation coefficient (r) between iFR and failed grafts was $0.332(\mathrm{p}=0.035)$. Graft failure was detected in three grafts $(8.1 \%)$ for group 1, in two grafts $(8.3 \%)$ for group 2, and in four grafts (16\%, all arterial grafts) for group 3. Statistically significant differences were found between groups 1 and $3(\mathrm{p}=0.041)$ and between groups 2 and $3(\mathrm{p}=0.044)$. No significant differences were found between groups 1 and $2(\mathrm{p}=$ $0.228)$.
\end{abstract}

Conclusions: The degree of coronary artery stenosis measured by iFR is a risk factor for attached graft failure. In a coronary artery where the iFR was haemodynamically non-significant, a higher rate of graft failure was detected.

Keywords: coronary artery bypass grafting; instantaneous wave-free ratio; graft failure, computed tomography angiography 